\title{
The Application of Integrated Traditional Chinese and Western Medicine in Anorectal Diseases
}

\author{
Yuyan Liu ${ }^{1,2, a^{*}}$ \\ ${ }^{1}$ Henan Province Hospital of Traditional Chinese Medicine, 450002.Zhengzhou, 450000, Henan, P. \\ R. China \\ ${ }^{2}$ The Second Affiliated Hospital of Henan University of Traditional Chinese Medicine, 450000, \\ Henan, P. R. China \\ aliuyuyan999888@163.com \\ * The Corresponding author
}

Keywords: Chinese medicine; Anorectal surgery; Western Medicine; Chinese Medicine; Situation

\begin{abstract}
Chinese medicine anorectal surgery is a more special subject, because some of the traditional Chinese medicine theory is more abstract, and the clinical teaching time is urgent, the teaching process is facing the patient's privacy problem, so the Chinese anorectal disease in the entire clinical treatment faced some difficulties. In this case, how to improve the clinical quality of anorectal surgery, the success of relevant personnel concerned about the key issues, this article will be the background of Chinese medicine characteristics of anorectal clinical teaching related content analysis. Analysis on the Current Situation of Clinical Teaching in Chinese and Western Medicine

Integrators of traditional Chinese and Western medicine in clinical practice often have many confusion, and even some loss or at a loss, more students in clinical practice and the lack of teachers and patients of the exchange ${ }^{1}$. As we all know, school medicine is difficult, difficult to learn Chinese medicine, Western medicine is even more difficult. Therefore, the Yuan Dynasty famous doctor Wang $\mathrm{Gu}$ wrote the title of Chinese medicine called "at this time is difficult." But once proficient in medicine, you can be a ten, a pass, like the Qing Dynasty famous doctor Chen Xiuyuan wrote the book "medicine is easy." For the clinical teaching of the anorectal, many students do not know how to grasp the key points of the class, the so-called focus of what we say, students often exist before the class is not a good preview and review the anorectal special physiological, pathology, And the etiology, pathogenesis, syndrome differentiation, treatment and prescription of each disease. In short, refers to the clinical teaching of many students with the most basic Chinese medicine, Western medicine are not ready to basics, to class, and clinical teaching effect can be imagined. In addition, in the clinical teaching of the anorectal, the communication between the doctor and the patient, the patient's examination is very important ${ }^{2}$. For example, perianal abscess, hemorrhoids disease preoperative and postoperative examination, dressing, doctors and patients need a good communication, so that patients have full confidence in the doctor, dressing skills are very critical, A certain skill. And these clinical skills can not be learned overnight, but not one or two classes can be grasped, you need students in the class to continue to pondering and taste the teacher in the clinical teaching classroom and patients and patients with family communication, patient examination Every detail.
\end{abstract}

\section{Traditional Teaching Mode}

China's traditional teaching model is the teacher spoon-type explanation, and students of mechanical records, this passive inculcation of teaching methods, the lack of appropriate interactive links, which just entered the clinical interns who is not difficult to see: you explain Things can be completed on time, you do not tell things will be difficult to find. For the traditional teaching model, the purely theoretical classroom teaching is possible, but the clinical teaching is the practice of doctors into clinical training clinical thinking of the critical period, not only medical theory, during which also contains the humanities, social, psychological, etc ${ }^{3}$. Many aspects in the clinical practice of traditional Chinese and Western medicine, only the use of traditional teaching model is not 
appropriate or is not perfect.

\section{Clinical Teaching of Chinese and Western Medicine for the Anorectal Clinical Teaching}

I usually pay more attention to fun and practical combination, so that students in the interest on the basis of memory, the choice of drug function on the more accurate. As we all know, rhubarb, betel nuts taste medicine is the most commonly used in the anorectal Chinese medicine, for the memory and use of rhubarb, the author usually in the clinical teaching class, so share with the students: Polygonatum Polygonaceae palm leaf rhubarb, Or rhubarb rhizome, because of this product color yellow, named rhubarb; and because of its function innovation, very Jun fast, so there are "generals" of the number. Rhubarb is mainly produced in Sichuan, Gansu, and Qinghai, Tibet and Yunnan and other places, where the clinical experience of the old Chinese medicine, the origin of rhubarb quite appreciated. Use rhubarb is to pass under the so, it is best to have the ability to achieve the purpose. Rhubarb bitter cold is diarrhea catharsis medicine, detoxification top grade. If it is taste tonic or longevity Dan, probably make the world stunned. Eastern Han philosopher and health scientist Wang Chong (AD 27 to about 97 years) said: want to longevity, intestinal often clear: to die, the intestines without slag. Jin Yuan famous doctor Zhu Danxi has put forward, That is, with a smooth stool to clear the intestinal toxins, Motherland medicine believes that intestinal toxins can lead to premature aging, but also lead to diseases, such as: loss of appetite, stomach fullness, nausea belching, bad breath, dizziness, headache, depression, and even induced gallstones, hemorrhoids, blood in the stool, cancer. Betel nut fruit contains a variety of nutrients and beneficial substances such as fat, betel nut oil, alkaloids, catechins, choline and other ingredients. Betel nut has a unique imperial malaria function, is the history of treatment of fruit, there are washing miracle Dan alias, physician Li Shizhen in the Compendium of Materia Medicain the record, betel nut under the edema, through joints, spleen tune center pain accumulation "And many other diseases. Not only that, betel nuts also cure glaucoma, intraocular pressure increased the effect of insect repellent embolism and anorectal common disease, frequently disease, the author usually share with the students: such as hemorrhoids and anal fissure difference, hemorrhoids is something prominent, With hemorrhagic pain, but the amount of bleeding hemorrhoids and bleeding frequency and hemorrhoids grading is inversely proportional to the performance of anal fissure can be summed up in one sentence: the smallest lesions, the largest pain, the most easily diagnosed, and anal fissure pain was cyclical. Other symptoms such as perianal abscess are red, swollen, hot, pain and dysfunction. Students just remember that a few, in the face of perianal abscess, you can immediately give diagnosis and treatment. We all know that Chinese medicine in the lock anal hemorrhoids, that is, anorectal cancer, it is our anorectal disease, serious illness, misdiagnosis rate is very high, the main reason is that clinicians ignore the anal doping, Palpable mass, can reach its size, location, activity and distance from the anus, but there is a word can be roughly summarized as anus referral: touch such as crater, rugged, you can doubt, should be asked to do further Colonoscopy. Most of the anorectal is a hot and humid type of betting, in fact not even, such as hemorrhoids we have hot and humid bets, but also in the gas shortage, qi stagnation and blood stasis embolism, Chinese medicine requires syndrome differentiation, it should be dialectical due to symptomatic treatment ${ }^{4}$.

\section{To Achieve Modern Teaching Methods and Chinese Medicine Anorectal Teaching Combination}

To Carry out Network Live Teaching. In the current clinical teaching, in order to ensure the quality of Chinese medicine anorectal surgery can achieve the desired, in the teaching process to actively use modern teaching methods, and constantly improve the quality of teaching to ensure that teaching results can be expected. For example, the promotion of live platform technology for modern mechanics teaching provides a new medium, colleges and universities to actively carry out network live teaching, significantly improve the teaching level. Therefore, this paper believes that the characteristics of Chinese medicine in anorectal clinical teaching, the need for scientific use of LAN-based network broadcast platform technology, and continuously improve the quality of 
teaching. To mixed hemorrhoids, for example, before the network live teaching, should first inform all students to start the network live time, and asked students to watch the network live in a timely manner. To the network broadcast platform teaching stage, the lecturer is entirely based on the Chinese medicine hemorrhoids to explain, rely on live movies, images, etc., to further explain the contents of the mixed hemorrhoids, so that all students can have a basic understanding of mixed hemorrhoids. For students in the course of learning, students can interact with the lecturers through the barrage, and the lecturer can quickly grasp the student's questions and answer them by observing the historical data. At the same time, the student's participation in the barrage can be the evaluation of student learning the key indicators, included in the internship results.

Compared with the traditional teaching methods, because the internship students for 90, 95, so the network broadcast this new teaching model has a higher degree of novelty, and then threw himself into the study, to ensure the quality of teaching. At the same time, such as mixed hemorrhoids these special body parts of the organ, through the LAN network broadcast mode to avoid the patient embarrassing emotions, affect compliance, but also help improve the quality of teaching ${ }^{5}$.

To Carry out Multimedia Teaching. Multimedia teaching has been widely used in the current clinical teaching of the hospital, becoming the main method of clinical teaching, and its teaching effect has been affirmed by many scholars. Therefore, in the teaching work, we must attach great importance to the study of multimedia teaching methods to further improve the quality of teaching throughout the teaching process, through the multimedia technology to patients from the clinical symptoms

To the classroom, so that students can learn the contents of the classroom have a correct understanding. At the same time, this method of visualization of the boring problem will further narrow the distance between the student and the clinic, and ultimately better meet the clinical requirements. At the same time in the multimedia teaching, to carry out WeChat teaching is also a new teaching method. In the teaching process, all students involved in clinical practice need to be set up in a WeChat masses, lecturers regularly distributed to the group on the clinical treatment of Chinese medicine anorectal network link. In this way, students will be able to understand the clinical advice at any time, strengthen the overall level of learning.

Focus on Teaching After the Expansion of Training. In the clinical teaching work, you can carry out outreach training, the results of this clinical teaching summary, and evaluation of teaching quality. The main contents of the evaluation include: (1) the clinical teaching courses, how much do you master? (2) What do you think of this clinical teaching? (3) For this clinical teaching, you would like to know what? In the whole development training, not only to the clinical teaching content into practical results, but also need to rise to the theoretical height, from the perspective of theoretical study of clinical teaching content has a more clear analysis, so that students can learn to learn by analogy Cultivate their own learning initiative, and guide students to extend teaching outside the classroom.

This paper focuses on the related contents of clinical teaching innovation of anorectal medicine, and analyzes the clinical teaching contents of anorectal anatomy from many angles. From the analysis results, innovative teaching methods are to ensure the quality of clinical teaching anorectal key. Therefore, in the whole clinical teaching work, the lecturer must correctly understand the particularity of Chinese medicine teaching; find the factors that affect the quality of teaching, and strive to improve the teaching methods, in order to further improve the clinical quality of anorectal surgery lay the foundation.

\section{Conclusions}

Chinese medicine culture, both the heritage of Chinese medicine, belongs to the natural scie nce category, but also the traditional Chinese cultural heritage, has a rich national cultural c onnotation. Western medicine is rigorous medicine; in the surgical operation is currently the world's most advanced medicine. The combination of traditional Chinese and Western medi cine will bring more health care to human health. The future of anorectal Chinese and Wes 
tern medicine clinical teaching is imperative will develop better and better. Only hope that every clinical teaching teachers can be more from the student point of view, the use of int egrated thinking of traditional Chinese and Western medicine to each anorectal disease can speak more vivid and more distinctive, so that students like, so in the clinical process of te aching, Students can really learn from the clinical teaching of clinical knowledge, master th e clinical skills.

\section{References}

[1] Chen HY, Jin XW, Li BR, et al. Cancer risk in patients with Peutz-Jeghers syndrome: A retrospective cohort study of 336 cases. Tumour Biol. 2017;39(6):1010428317705131.

[2] $\mathrm{Wu} \mathrm{S}$, Yuan W, Shen Y, et al. The miR-608 rs4919510 polymorphism may modify cancer susceptibility based on type. Tumour Biol. 2017;39(6):1010428317703819.

[3] Peng J, Lin J, Qiu M, et al. Oncogene mutation profile predicts tumor regression and survival in locally advanced rectal cancer patients treated with preoperative chemoradiotherapy and radical surgery. Tumour Biol. 2017;39(7):1010428317709638.

[4] Park EJ, Yoon YT, Hong CK, Ha YR, Ahn JH. Randomized, noninferiority study between video versus hand ultrasound with wet foam dressing materials to simulate B-lines in lung ultrasound: A CONSORT-compliant article. Medicine (Baltimore). 2017;96(30):e7642.

[5] Dong Y, Liu Y, Shu Y, et al. Link between risk of colorectal cancer and serum vitamin E levels: A meta-analysis of case-control studies. Medicine (Baltimore). 2017;96(27):e7470. 